\title{
Mit Pflanzenkraft zu schöner Haut und glänzenden Haaren
}

\section{Die Firma RAUSCH AG KREUZLINGEN stellt sich vor. $R$. Höhl}

Wir freuen uns, heute ein Gespräch mit Marco Baumann, Al leineigentümer und Verwaltungsratspräsident der RAUSCH AG ratspräsident der RAUSCH AG
KREUZLINGEN, führen zu dürfen.

Promed komplementär: Ihre Firma hat eine lange Tradition. Seit wann besteht sie?

BAUMANN: Die Firma Rausch besteht seit 1890. Dieses Jahr feiern wir das 120-jährige Firmenjubiläum. Im Jahr 1900 hat Josef Wilhelm Rausch die erste medizinische Kopfwaschseife, mit damals pH 8,5, auf den Markt gebracht, konfektioniert in einer Braunglasflasche.

Worauf sind Sie spezialisiert, wo liegt Ihre Stärke?

BAUMANN: Ganz eindeutig: Her Rausch hatte das Kräuterwissen empirisch vom großen Fundus der Klöster übernommen. Abgeleitet davon stellte er selbst Kräutersäfte, Extrakt und Tinkturen her; 1919 entstanden die erste flüssige, medizinische TeerSeife und ein antiseptisches CamillenShampooing. Er starb 1935. Mein Vater erwarb im Oktober 1949 die Firma und lancierte, aufgrund seiner langjährigen Branchen-Erfahrung, 1951 das erste alkalifreie Kräuter-Shampoo „Exalkali“. 1955 folgte die erste Haarpackung in der Schweiz, mit 20 Prozent Zinnkrautextrakt. Das haben wir bis heute beibehalten: Wenn Sie heute eine RAUSCH Zinnkraut Repair-Packung applizieren, wirkt $20 \%$ reines Zinnkraut, zum Wohle von
Wo wachsen die Kräuter, welche Ihren Rezepturen zugrunde liegen? BAUMANN:Wirhaben in derSchweiz fünf Vertragsbauern, die aus kontrolliertem Anbau die Kräuter liefern. rer Produktion. Was in der Schweiz nicht gedeiht, kaufen wir ein - aus frischen Ernten des Weltmarkts. So hat es sich ergeben, dass wir aus Brasilien Öle beziehen - aus ganz speziellen kostbaren Nüssen, wie z. B. aus Brasilianischen Kastanien und Macadamia, um einige zu nennen.

Die Entwicklung von Haar- un Hautproblemen ist weltweit steigend. Als Spezialitätenmarke in der Nisch ist es heutzutage notwendig, die Konsumenten möglichst gut aufzuklären und zu sensibilisieren, naturnahe Präparate für ihr jeweiliges Problem zu verwenden. Einige Wirkstoffe, welche uns die Natur nicht zur Verfügun stellt, bzw. wo wir noch nicht dahinte gekommen sind, diese herauszufinden, substituieren wir mit einem Minimum an Chemie. Dazu stehen wir.

Wir streben danach, möglichst alle Prozesse in unserem Stammhaus durchzuführen. Von 58 Kräutern, die wir verwenden, werden 42 im Haus sorgfältig nach hauseigenen Verfahren extrahiert.

Haben Sie persönlich eine Bezie hung zu Pflanzen und Kräutern? BAUMANN: Ja, sicher. Meine Beziehung zu Kräutern entstammt meine Kinder- und Jugendjahren. Mein Vater beauftragte mich oft, Kräuter zu jäDies ist ein wichtiges Element unse- entsorgen, haben wir auf Porzellan und Glas umgestellt, das mit einer Geschirrspülmaschine gewaschen und wieder verwendet wird. RAUSCH war übrigens die fünfte Firma in de Schweiz, die bereits in den Achtzige Jahren mit einer Ökobilanz gearbeite hat. Und unsere Druckereien kontrollieren wir hinsichtlich der Papiersoren sowie der Cadmiumbelastung in den Druckfarben, um nur einige Beipiele zu nennen. Viele solcher Aktiviäten sind längst Standard bei uns. Wi sind ISO-zertifiziert und produziere nach GMP-Richtlinien.

Welche Schlagworte charakterisieren die RAUSCH Produkte?

BAUMANN: Seit jeher verzichte RAUSCH auf Tierversuche. Mit unsere GCMS-Maschine (Massenspektrometrie) können wir Messungen auch im führen. Unsere Produkte sind bodenständig, solid, bewährt - Produkte, di mit Natürlichkeit bei bester Verträglichkeit nachhaltige Resultate bringen, zum Nutzen der Konsumenten.

An welche Konsumenten wenden Sie sich?

BAUMANN: An Personen, die naturnahe Produkte bevorzugen, oder sol che mit Haarproblemen. In der Regel sind Frauen unsere Käuferinnen, viele z.B. mit Kopfhautproblemen, auch nach einem Kindbett oder nach einer Chemotherapie.

Wir arbeiten mit Extrakten, die, ähnlich wie guter Wein, über Monate Ökobereich auf den Trilliardstel durch-

Unsere Produkte sind verträglich und sicher. Auch Personen mit hohem Allergiepotential können sie zum größten Teil problemlos verwenden.

Apotheker sind Ihre Partner: Si haben in Deutschland als beste Partner in der Haarpflege im Jah 2010 zum 7. Mal in Folge die Goldmedaille erhalten. Werden Ihre Produkte ausschließlich über Apotheken vertrieben?

BAUMANN: Es ist uns wichtig, das der Kunde richtig beraten wird. Daher schätzen wir die langjährige $\mathrm{Zu}$ sammenarbeit mit den Apothekern und dem spezialisierten Fachhandel. In Korea und China arbeiten wir mi einem anspruchsvollen Shop-in Shop Konzept

Streben Sie auch eine Zusammen arbeit mit Ärzten an?

BAUMANN: Ja, im Besonderen di Zusammenarbeit mit Dermatologen.

Der Kunde schätzt die Produkte und gibt vermutlich auch gerne etwas mehr aus, wenn er weiß, das die Qualität stimmt.

BAUMANN: Ja, das ist richtig. Wir haben vor etwa acht Jahren eine Untersuchung zum Thema Kundentreue gemacht. Es zeigte sich ein interessantes Resultat: Über 40 Prozent de RAUSCH-Shampoos werden übe den Zeitraum von länger als drei Jahren regelmäßig von denselben Personen gekauft. In einer anderen Studie der IHA Schweiz steigen Frauen ent-
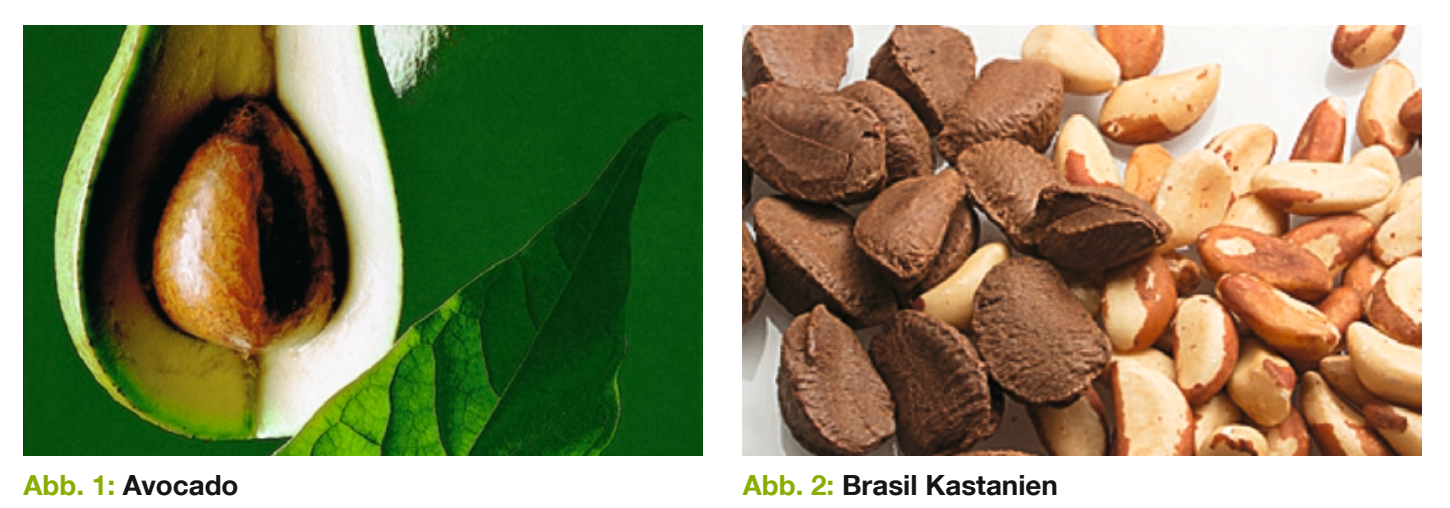

Abb. 2: Brasil Kastanie

Abb. 1: Avocado

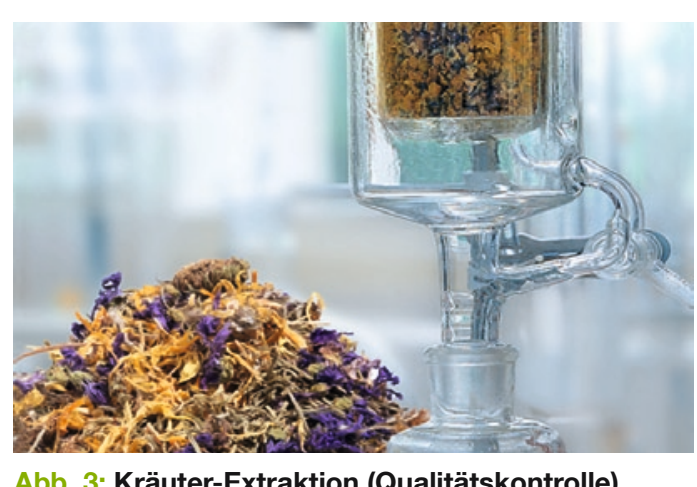

Kopfhaut und Haar, weil es beachtliche Mengen an Kieselsäure enthält.

Viele Menschen leiden heute unte Haar- und Kopfhautproblemen; juckende Kopfhaut, Schuppen, strapaziertes Haar-z. B.im Zusammenhan mit Colorationen oder minderwertigen Produkten. Unsere Philosophie in dieser Sache: Erstens, wir sorgen für eine gesunde Kopfhaut und tragen dazu bei, auch die Haare gesund zu erhalten. Zweitens, wir sind kompetent, jede Art von Haar- und Hautproblem zu lösen und Nutzen zu bieten.

Die Rausch Kompetenz hat sich vom Empirischen zum Wissenschaftlichen entwickelt: Dies begann vor etwa 35 Jahren, als mein Vater ein pH-Meter in die Herstellung einbrachte. Heute gibt es einen umfassenden, wissenschaftlich ausgearbeiteten und kontrollierten Haarund Körperpflegeplan. ten oder zu sammeln. 1968 bin ich dann definitiv in die Firma RAUSCH eingetreten.

Wo ist Ihr Firmenstandort? BAUMANN: In Kreuzlingen, am Bodensee - in der Schweiz

Praktisch 70 Prozent aller Kosmetika werden heute durch Drittherstelle d.h. durch Lohnhersteller, gefertigt. Dadurch sind im Massenmarkt viele Produkte austauschbarer geworden. Bei uns gibt es lediglich zwei Ausnah men: eine Sterilabfüllung für Körpe creme und die Aerosolabfüllung.

Ist Ökologie ein Thema?

BAUMANN: Ökologie ist ein ganz wesentliches Thema für RAUSCH:Unser Firma hatte den ersten VW-Bus mit Katalysator in der Schweiz. Und wir brauchen keine Plastikbecher mehr: Statt 43.000 Plastikbecher pro Jahr zu und kostet auch Zeit. Dass die Preise unserer Produkte etwas höher sind als jene der industriell gefertigten liegt daher auf der Hand.

Seit 1890 gibt es RAUSCH in der Schweiz, seit 1971 sind RAUSCH Produkte auch in Österreich auf dem Markt. Inzwischen ist RAUSCH in 26 Ländern vertreten. Ich mache die Schulungen zum Großteil selbst. In Hongkong sind wir akkreditiert und haben dort bereits zum zweiten Ma kosmetika erhalten.

RAUSCH Produkte sind strengsten Kontrollen unterworfen. ISO-zertifiziert und nach GMP-Richtlinien zu Kontrollen einen fixen Aufwandposten darstellen. Jeder Prozess ist sauber beschrieben. Das heißt, wir sind kompetent und sorgen für Qualität reifen müssen. Dies ist aufwändig die höchste Auszeichnung für Haararbeiten bedeutet, dass lückenlos weder nach dem ersten Baby oder nach dem zweiten Jobwechsel al RAUSCH Kundinnen ein.

Noch eine weitere Erkenntnis: Wenn es Frühling wird, machen viele Frauen eine Diät - und ca. zehn Wofall. Grund: Durch Nährstoffmange kann es zu Haarausfall kommen. Dies gab den Anstoß für die Entwicklung unseres Nahrungsergänzungsmittels RAUSCH Schweizer Kräuter Vital Kapseln. Es handelt sich um ein Kombination von jeweils zwei Kapseln, mit dem einzigartigen „DUO active“ Wirkprinzip: Die Öl-Kapsel enthält drei hochwertige Öle: GoldhirsePerilla- und Algen-Öl, reich an hoch ungesättigten Fettsäuren, sowie die Vitamine $\mathrm{A}$ und $\mathrm{E}$ für einen gesunden Stoffwechsel der Haut. Die KräuterKapsel beinhaltet einen Grüntee-Extrakt der reinsten Qualität (OM24) chen späterklagen vieleüber Haaraus-

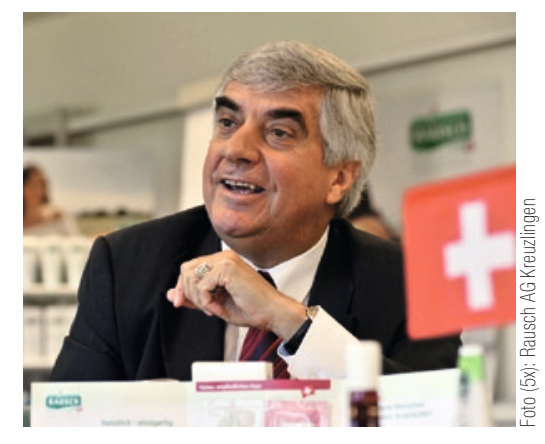

\section{Marco Baumann} Alleineigentümer und Verwaltungsratspräsident der

„Es gibt den einzigartigen Unterschied, und für Natur gibt es keinen Ersatz.“

sowie eine spezielle Schweizer Kräutermischung als natürliche Radikalfänger. Zudem unterstützen der Vitamin B-Komplex, Biotin, Pantothensäure und Zink das Wachstum gesunder, kräftiger Haare und Nägel. Insgesamt enthalten RAUSCH Schweize Kräuter Vital Kapseln 23 hochwertige Wirkstoffe. Die zwei Kapseln sollen $1 \mathrm{x}$ morgens, nach dem Frühstück, eingenommen werden.

Gibt es Produkte in Ihrer "Pipeline über die Sie uns bereits ein wenig Information geben können?

BAUMANN: Ich möchte noch nicht viel darüber verraten. Es wird wieder ein Produkt zur Unterstützung der Gesundheit und Schönheit sein. 